\title{
RESEARCH ANNOUNCEMENTS COVERING SMOOTH HOMOTOPIES OF ORBIT SPACES
}

\author{
BY GERALD W. SCHWARZ ${ }^{1}$ \\ Communicated by P. T. Church, April 5, 1977
}

1. Introduction. Let $G$ be a compact Lie group. If $M$ is a $G$-space, then one may consider $M$ as a sort of singular fiber bundle over the orbit space $M / G$. Palais has proved a covering homotopy theorem and constructed classifying spaces for such types of "bundles" [8]. In this note we describe an analogue of Palais' covering homotopy theorem which is valid for smooth $G$-actions. Various guises of this smooth Palais theorem have already been important in the study of regular actions of the classical groups (see [3], [4], [5] and references therein).

2. Orbit spaces. Let $X$ and $Y$ be smooth $\left(=C^{\infty}\right) G$-manifolds. A real-valued function on $X / G$ is said to be smooth if it lifts to a smooth function on $X$, so $C^{\infty}(X / G) \simeq C^{\infty}(X)^{G}$. F:X/G $\rightarrow Y / G$ is said to be smooth if $F^{*} C^{\infty}(Y / G) \subseteq$ $C^{\infty}(X / G)$. By the slice representation at $x \in X$ we mean the representation of the isotropy group $G_{x}$ on the normal bundle at $x$ to the orbit $G x$. Two $G$-orbits are said to have the same normal type if they contain points with the same isotropy group and isomorphic slice representations (up to trivial factors). The subsets of orbits of given normal type give a stratification of $X / G$ by $C^{\infty}$ manifolds. One can show that $X / G$ is locally homeomorphic to closed semialgebraic sets where the homeomorphisms can be chosen to induce isomorphisms on rings of $C^{\infty}$ functions and, up to components, to preserve strata ([1], [9]). Here a closed semialgebraic set is given its canonical stratification by singularity and the ring of $C^{\infty}$ functions induced from the ambient space.

If $\xi \in X / G$, let $T_{\xi}(X / G)$ denote the Zariski tangent space at $\xi$, i.e. the dual of $M_{\xi} /\left(M_{\xi}\right)^{2}$ where $M_{\xi}$ is the maximal ideal of the ring of germs of smooth functions at $\xi . T_{\xi}(X / G)$ is always finite dimensional. Let $\sigma_{\xi}$ denote the stratum of $X / G$ containing $\xi$, and let $N_{\xi}(X / G)$ denote the normal space $T_{\xi}(X / G) / T_{\xi}\left(\sigma_{\xi}\right)$. Der $C^{\infty}(X / G)$ denotes the real-linear derivations of $C^{\infty}(X / G)$, and we call elements of Der $C^{\infty}(X / G)$ smooth vector fields on $X / G . B \in \operatorname{Der} C^{\infty}(X / G)$ is said to be strata preserving if for each $\xi \in X / G$ the associated tangent vector $B(\xi)$ lies in $T_{\xi}\left(\sigma_{\xi}\right) . X^{\infty}(X / G)$ denotes the strata preserving elements of $\operatorname{Der} C^{\infty}(X / G)$, and $X^{\infty}(X)$ denotes the smooth vector fields on $X$.

AMS (MOS) subject classifications (1970). Primary 57E15, 58C25.

Key words and phrases. Covering homotopy theorem, transformation group, stratification, normal orbit type, normally transverse map.

1 Research partially supported by NSF Grant \#MCS 75-8555 A01. 
A smooth strata preserving map $f: X / G \rightarrow Y / G$ is said to be normally transverse if the differential of $f$ induces an isomorphism of $N_{\xi}(X / G)$ with $N_{f(\xi)}(Y / G)$ for all $\xi \in X / G$.

3. Main results. The following is our smooth version of Palais' covering homotopy theorem.

THEOREM I. Let $f: X \rightarrow Y$ be an equivariant smooth map such that the induced map $\bar{f}: X / G \rightarrow Y / G$ preserves normal type. Suppose that $\bar{F}: X / G \times$ $[0,1] \rightarrow Y / G$ is a smooth strata preserving homotopy starting at $\bar{f}$ such that $\bar{F}(\cdot, t): X / G \rightarrow Y / G$ is normally transverse, $0 \leqslant t \leqslant 1$. Then there is a smooth equivariant homotopy $F: X \times[0,1] \rightarrow Y$ inducing $\bar{F}$ and starting at $f$. Moreover, any two such liftings of $\bar{F}$ differ by a smooth equivariant isotopy of $X$ which starts at the identity and induces the trivial isotopy on $X / G$.

Restricted versions of Theorem I are in [1], [2], and [4]. It is not difficult to reduce Theorem I to the special case of covering smooth isotopies of $X / G$. Such isotopies are obtained by integrating time-dependent strata preserving vector fields, and Theorem I follows easily from the first part of

THEOREM II. (1) The natural map $\pi_{*}: \mathfrak{X}^{\infty}(X)^{G} \rightarrow \operatorname{Der} C^{\infty}(X / G)$ has image $\mathfrak{X}^{\infty}(X / G)$.

(2) There is a direct sum decomposition

$$
\mathfrak{X}^{\infty}(X)^{G}=\operatorname{Ker} \pi_{*} \oplus W
$$

where $W$ is a closed subspace of $X^{\infty}(X)^{G}\left(C^{\infty}\right.$ or Whitney topology).

The differentiable slice theorem (see [2]) allows us to reduce the proof of Theorem II to the case of representations. One can then further reduce to proving an algebraic analogue of (1) which we establish using techniques from algebraic geometry and invariant theory. Main ingredients are Luna's slice theorem [6] and the Hilbert-Mumford criterion [7, Chapter 2].

During this work I have benefited from conversations with many mathematicians, and I would especially like to thank E. Bierstone, D. Buchsbaum, D. Eisenbud, M. Hochster, D. Lieberman, and J. Mather.

\section{REFERENCES}

1. E. Bierstone, Lifting isotopies from orbit spaces, Topology 14 (1975), 245-252. MR 51 \#11551.

2. G. E. Bredon, Introduction to compact transformation groups, Academic Press, New York, 1972.

3. - Biaxial actions, Mimeographed Notes, Rutgers University, 1974.

4. M. Davis, Smooth actions of the classical groups, Thesis, Princeton University, 1974.

5. - Smooth G-manifolds as collections of fiber bundles (to appear).

6. D. Luna, Slices étales, Bull. Soc. Math. France, Mémoire 33, Soc. Math. France, 1973, pp. 81-105. MR 49 \#7269. 
7. D. Mumford, Geometric invariant theory, Springer-Verlag, Berlin and New York, 1965. MR $35 \# 5451$.

8. R. S. Palais, The classification of G-spaces, Mem. Amer. Math. Soc. No. 36, 1960.

9. G. W. Schwarz, Smooth functions invariant under the action of a compact Lie group, Topology 14 (1975), 63-68. MR 51 \#6870.

SCHOOL OF MATHEMATICS, INSTITUTE FOR ADVANCED STUDY, PRINCETON, NEW JERSEY 08540

DEPARTMENT OF MATHEMATICS, BRANDEIS UNIVERSITY, WALTHAM, MASSACHUSETTS 02154 